\title{
On the use of tapered channels gas distributors to promote convection in PEM Fuel Cells
}

\author{
Alessandro d'Adamo ${ }^{1, *}$, Massimo Borghi ${ }^{1}$ \\ ${ }^{1}$ Department of Engineering “Enzo Ferrari”, University of Modena and Reggio Emilia, Via Vivarelli \\ 10, Modena 41125, Italy
}

\begin{abstract}
Polymeric Exchange Membrane Fuel Cells (PEMFC) are promising power propulsion systems for the decarbonization of the transportation sector. Despite being a well-known method for the direct production of electric current from the reactants chemical energy, one of the major limitations to their large-scale industrial development are fluid dynamics and mass transport aspects, crucially limiting the electrochemistry rate under critical conditions. This is especially verified in PEMFC with serpentine-type gas distributors, for which such areas are identified in proximity of the gas channel bends where the dominant mechanism for species transport shifts from a convection-enhanced to a diffusion-limited one. An engineering method to enhance the convective transport in such deficient areas is the use of gas distributors with tapered channels, effectively forcing the flow in diffusive media and improving the reactants delivery rate and products removal. A numerical analysis is presented on a limited domain representing a section of a serpentine gas distributor. A multi-dimensional CFD study is carried out comparing conventional-type and tapered channel distributors, evaluating the combined effect of pressure losses, catalyst layers utilization, flow regime in anisotropic diffusion media and convection/diffusion balance via a non-dimensional analysis. The study covers various inlet Reynolds numbers and in-plane permeability of porous materials for two diffusion media thicknesses, with the aim to extend the generality of the study. Conclusions based on the simulation results outline channel tapering as a very effective way to improve the power density of PEMFC, although an energetic cost/benefit analysis indicates a reduced cell efficiency.
\end{abstract}

\section{Introduction}

Fuel cells are considered one of the most promising technologies for a sustainable power generation, being able to produce electric power via electrochemical reactions. This avoids the issues related to a combustion-based system, both from a thermodynamic (Carnot cycle as upper limit to the theoretical efficiency) and an emission (pollutants formation) standpoint $[1,2]$, whose development has been furthered by the development of advanced numerical models proposed in the recent years [3-5]. In the transportation sector, Polymeric Exchange Membrane Fuel Cells (PEMFC) are the most promising fuel cell type, thanks to high efficiency, absence of corrosive electrolyte, low-temperature operation, and rapid start-up time. In such application it is of utmost importance to maximize the cell power density to reduce weight and volume of the power system. This motives the engineering focus on high

\footnotetext{
* Corresponding author: alessandro.dadamo@unimore.it
} 
current density operations, which I) are characterized by the maximum electrochemical reaction rate, II) suffer from the maximum waste heat generation and the maximum liquid water formation rate, and III) are limited by the reactants concentration at active Catalyst Layers (CL), also known as "concentration overpotential". In the past decades, several configurations for Gas Channels (GC) and Gas Diffusion Layers (GDL) were proposed to suppress (or limit) the concentration overpotential, hence effectively promoting the cell power density and designing attractive solutions for the mobility sector. Li et al. [6] reviewed the multiple and diverse types of GC, pointing out pros and cons for each and underlying the reasons for the prevailing serpentine-type geometry, i.e. better reactants distribution and water removal capacity, despite higher pressure losses. Similar conclusions were experimentally drawn by Taccani et al. [7] for straight-parallel, 4-step and 5-step serpentine PEMFC. In [8] Pharoah numerically investigated the pressure losses and flow distribution in a simplified serpentine domain, pointing out the relevance of including realistic non-isotropic GDL permeability, representing the resistance for the flow by-pass and often overlooked in multi-dimensional numerical simulations (i.e. assumed as isotropic). Feser at al. [9] reinforced the concept underlying the different nature of diffusive and convective transport processes: the former being a poorly controllable and inherently gradient-limited process (with diffusive flux $j_{\text {diff }, i}=-D_{i} \nabla Y_{i}$, with $D_{i}$ being the diffusivity coefficient and $Y_{i}$ the mass fraction of the $i$-th species), whereas the latter is an externally controllable process (with convective flux for the $i$-th species $j_{c o n v, i}=\tilde{\boldsymbol{u}} Y_{i}$, with $\tilde{\boldsymbol{u}}$ being the local mean velocity vector), suitable for engineering optimization and acting alongside the diffusive transport. Moreover, the concept of a beneficial under-rib flow through the porous GDL (as a consequence of the pressure differential between non-separated adjacent channels) was proposed in [9] for a more uniform current generation, indicating the need of an integrated GC/GDL design to favourable use this phenomenon. A known issue in traditional serpentine designs is the channel bend region, where the fluid continuity of gas channels nullifies the intra-channel $\Delta p$, leading to a poor reactants delivery at CL in such areas. This was extremized in [10] where a serpentine-type gas distributor was modified to minimize the number of adjacent channel communication (apart from GDL), thus ubiquitously creating a beneficial intra-channel $\Delta p$ for maximized surface usage. An extreme case of design variation to overcome such limitations is the interdigitated configuration [11], where all the flow is abruptly forced in the GDL using channel occlusions. The complex 3D nature of the flow and the relevant current density improvement show the design possibilities to guide the flow for optimized electrochemical reactivity, paving to way to the use of 3D-CFD models to tackle the limiting processes and exploring innovative yet geometrically simple variations. A more gradual way to induce flow by-pass is to design tapered GC, i.e. progressively reducing the flow area along the channel direction. Yan et al. [12] numerically investigated the effect of height and width tapering on PEMFC operation on a 3D isothermal single-phase model. They showed the effectiveness of a channel height reduction and/or width expansion in the flow direction: in the former case, the positive mechanism was the flow area contraction, forcing more flow in the porous GDL, whereas in the latter case the increased GC/GDL passage area allowed more flow to diffuse in the GDL. Both mechanisms led to delayed concentration losses and to higher power output, although no estimation of cell efficiency gain/loss was presented considering the varied pressure loss. A similar concept was numerically investigated by Chowdury et al. [13], where the benefit of a serpentine-type distributor was modified into a converged serpentine to promote in-GDL flow and reduce the concentration loss.

In this paper, a numerical study is presented on a simplified domain with the aim to evaluate both effectiveness and efficiency of tapered channels configurations for serpentine-type gas distributors. The modelling set was presented in [14], where the authors developed a nonisothermal, multi-dimensional and multi-phase model for the fluid-dynamic and 
electrochemical simulation of a straight-channel hydrogen-air PEMFC, which was applied to a serpentine-type PEMFC in $[15,16]$. Here, the three-dimensional flow of a serpentine-type PEMFC is simulated on a representative portion, with characterization of the flow by-pass ratio through the porous GDL. The choice of a height-only tapering effect is pursued, as width-tapering would result in varied contact area for heat/charge transport between GDL and Bi-Polar Plate (BPP) which would need to be separately considered.

\section{Numerical Model}

A sample section of a serpentine-type gas channel with porous GDL is considered in this study and it is reported in Figure 1. Given the focus on flow field analysis, the hypotheses of steady-state, laminar flow and ideal gases are adopted. The flow characterization is carried out without including electrochemical reactions, and the condition at the bottom GDL surface is considered as representative of flow at CL. Moreover, the solid BPP is not explicitly included, having the sole effect of flow guidance which is replicated in the fluid domain shape. The operating fluid is atmospheric air $\left(21 \%-79 \% \mathrm{O}_{2}-\mathrm{N}_{2} \mathrm{~mol} \%\right)$, at ambient pressure and $353 \mathrm{~K}$, representative of cathode fluid and flow. A macro-homogeneous approach is adopted for the porous GDL, consisting in applying global properties representative of a statistically representative material sample without the computational burden of explicitly meshing the porous structure of the solid phase. In this model, typical values of PEMFC GDL are applied, such as porosity $\varepsilon=0.7$ and Bruggeman-type tortuosity $\tau$ [17]. A fundamental characteristic of GDL is their permeability ( $K$, in $\left[\mathrm{m}^{2}\right]$ units), acting as a resistance for the incoming flow and included as a momentum source term in the form of Darcy's law (justified by the laminar low-velocity flow regime in such materials) [18]. Despite the still common use of isotropic $K$ for GDL modelling, it is well established that their layered composition of fibrous carbon papers induces a markedly anisotropic permeability. In this study this aspect is implemented in the model, distinguishing between a through-plane $\left(K_{\perp}=1 \times 10^{-12} \mathrm{~m}^{2}\right)$ and an in-plane permeability $\left(K_{\|}=1 \times 10^{-12} \sim 1 \times 10^{-9} \mathrm{~m}^{2}\right)$. In order to consider the bypass flow in the orthogonal direction than the GC axis, periodic boundary conditions are applied at GDL lateral sides (see Figure 1), and a velocity inlet and pressure outlet are applied. All the remaining boundaries are treated as adiabatic walls. The $3 \mathrm{D}$ domain is discretized with a structured hexahedral mesh, with conformal interfaces at GC/GDL contact. A reference cell size of $0.1 \mathrm{~mm}$ is adopted, with mesh refinement in the through-plane direction in the GDL (as low as $0.02 \mathrm{~mm}$ ). The total number of cells is approx. 520'000 for all cases, and SIMCENTER STAR-CCM+ 2020.2 licensed by SIEMENS DISW is used for the model creation and simulation run.

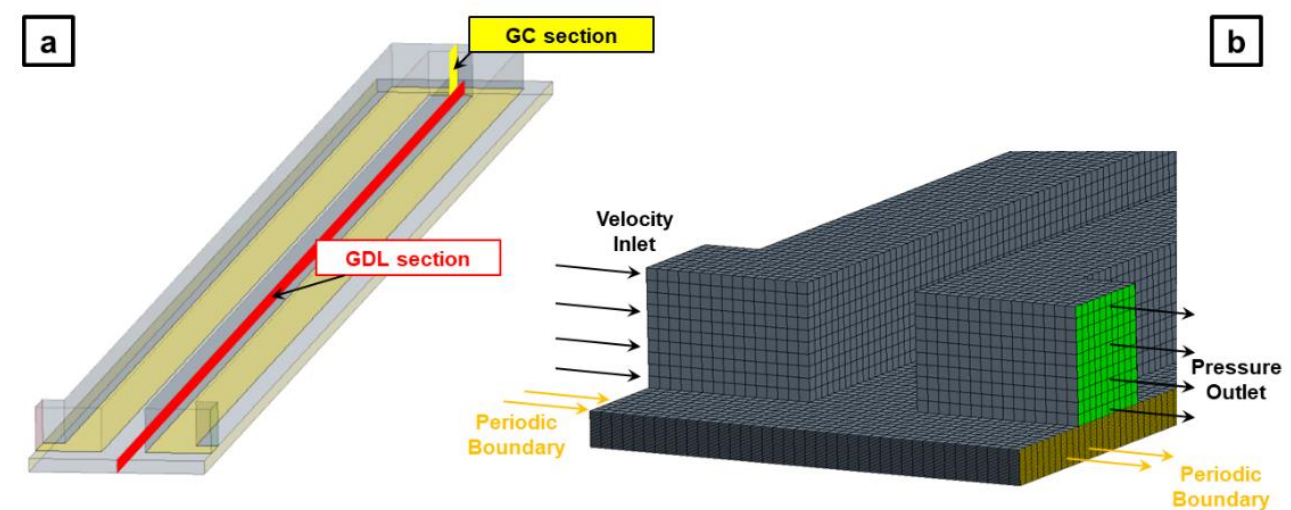

Fig. 1. Simulated domain with GC/GDL sections (a) and sketch of the numerical grid (b). 
Gas channel tapering is applied in the height direction, reducing the channel height from 1 $\mathrm{mm}$ (standard value for PEMFC channels) to $0.1 \mathrm{~mm}$ (called T01), hence using a tapering ratio of $10 \%$ from its nominal value with respect to the standard case (STD). In Figure 2 the channel tapering in the channel bend portion is visible, and it is designed to promote the flow in a well-known area of poor CL utilization. This is mainly motivated in the STD design by the reduced $\Delta p$ between adjacent channels in proximity of the GC bend, leading to GCpreferential flow path. Two GDL thicknesses $\left(h_{G D L}\right)$ are considered (300 and $125 \mu \mathrm{m}$, respectively), representative of typical thick/thin diffusion media, and for each $h_{G D L}$ standard and tapered GC are simulated, for a total of 4 investigated geometries. For every configuration, 3 flow conditions are analysed at inlet Reynolds number of $R e=100,200$ and 400 (from which the inlet velocity is calculated) on 7 levels of in-plane permeability $\left(K_{\|}\right)$for the porous GDL, for a total of 21 cases for each design.

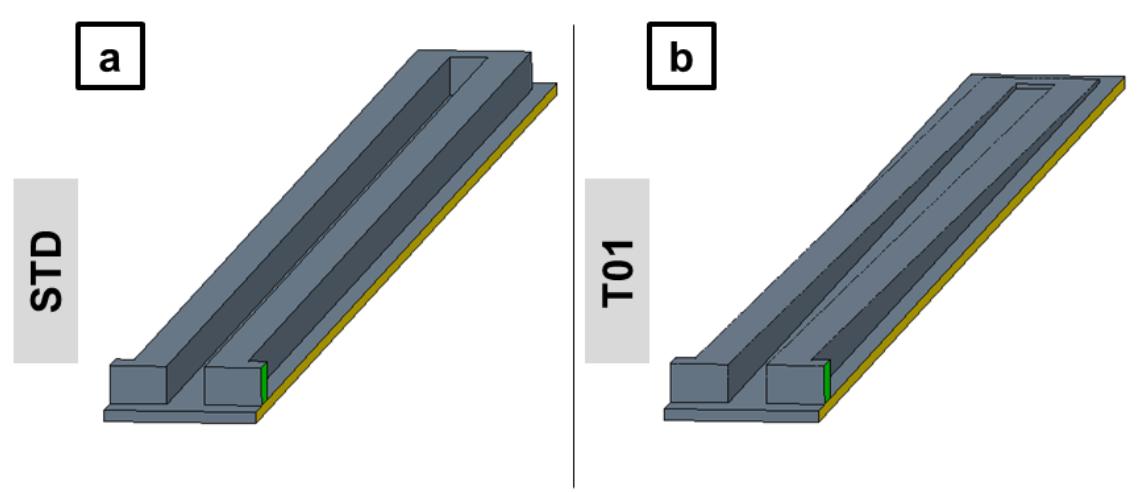

Fig. 2. Geometry of the standard (STD) (a) and of the tapered (T01) (b) design.

\section{Results}

The first observed results are the pressure losses and the flow ratio in the GDL (i.e. the entity of the channel by-pass) for all configurations. As for the former, the partial channel obstruction is explicitly designed to promote cross-flow through the porous GDL, hence a pressure loss increase is expected. The pressure differential between the outlet and the inlet sections is reported for all cases in Figure 3 (top). In the STD configuration, the pressure loss decreases for high- $K_{\|}$GDL and for low- $R e$ flows, whereas it is poorly sensitive to GDL thickness $h_{G D L}$. When channel tapering is introduced, $\Delta p$ systematically increases for all T01 configurations. The augmented pressure drop is tolerable only for high- $K_{\|}$GDL, which facilitate the forced flow passage through the GDL, but it exponentially grows for low- $K_{\|}$ GDL.

Regarding the flow distribution between GC and GDL, Figure 3 (bottom) shows that the flow percentage by-passing the GC for the STD case increases from being negligible (for the least permeable GDL) to approx. $40 \%$ of the total flow rate (for the most permeable GDL), while being insensitive to both $h_{G D L}$ and $R e$. 


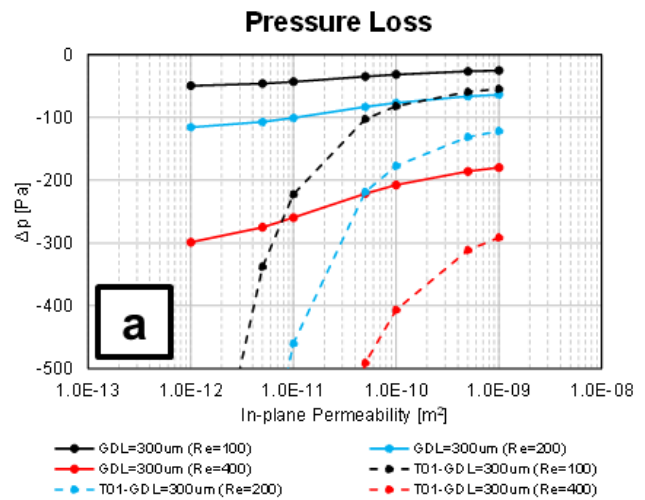

Flow Percentage in GDL

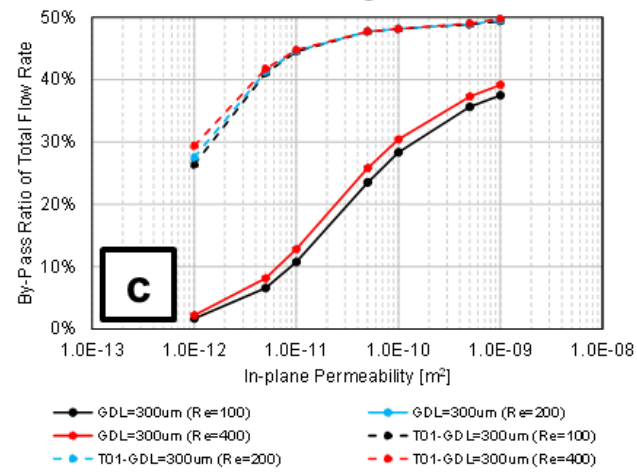

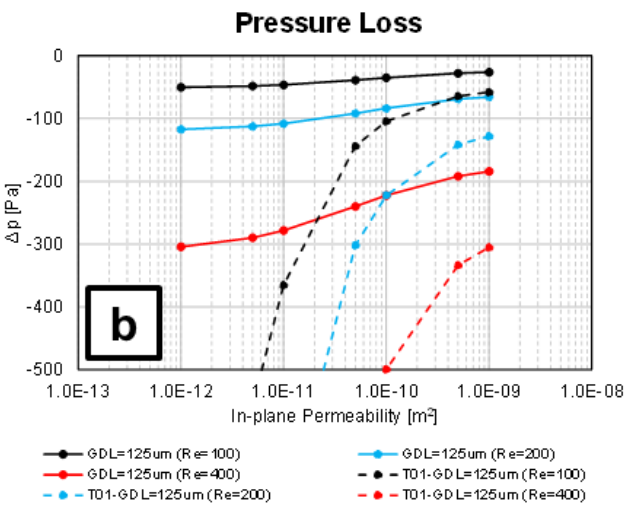

Flow Percentage in GDL

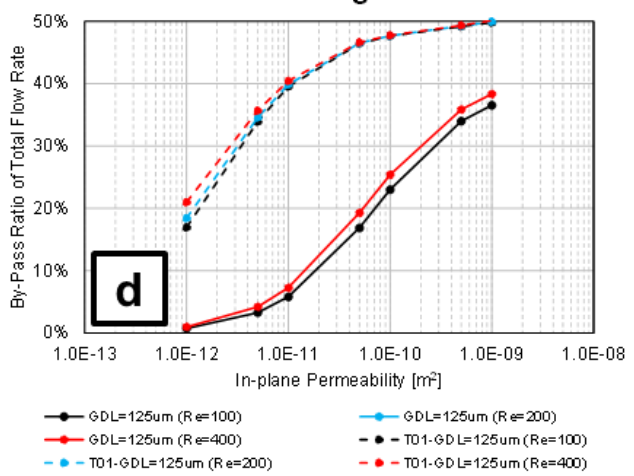

Fig. 3. Top row: pressure loss for the $300 \mu \mathrm{m}$ (a) and the $125 \mu \mathrm{m}$ (b) for all cases. Bottom row: flow percentage through the GDL section for the $300 \mu \mathrm{m}$ (c) and the $125 \mu \mathrm{m}$ (d) for all cases.

Tapered design introduces a progressive cross area obstruction, leading to a local pressure increase which is an additional force for flow by-pass through the GDL. Conversely, in the opposite channel the progressive area expansion promotes a depression and a suction action for flow from the GDL. The net effect is that pressure differential across adjacent channels is increased; this combines with the designed area reduction in the GC, both aspects increasing the flow percentage moving through the GDL. This ranges from $30 \%$ to $50 \%$ of the total flow rate for all cases in T01 design, and it is quantified on the two transverse sections reported in Figure 1 (a).

The tapered configuration is designed to promote GDL flow, despite the additional energy cost for pressure drop reported in Figure 3. Hence, the discussion moves to the flow regime in the GDL. The average flow velocity on a transverse section plane in the GDL largely increases with tapered designs, as reported in Figure 4 (top). This represents a drastic change in the flow regime and in the dominating transport mechanism: in addition to the ever-present diffusion-driven transport, an emerging convective-type transport grows for T01 designs. With the aim to quantify the dominating transport process, a modified Peclet number definition (Eq. 1) was introduced by Feser et al. [9] and here applied, where $b=1 \mathrm{~mm}$ is the land width, $D=3.2 \times 10^{-5} \mathrm{~m}^{2} / \mathrm{s}$ is the binary diffusivity of air, and $|\widetilde{\boldsymbol{u}}|$ the magnitude of the local mean velocity vector:

$$
P e=\frac{|\widetilde{u}| \cdot b}{\varepsilon \cdot D}
$$

In Figure 4 (bottom) the volume average $P e$ on the GDL is reported, showing that for all STD designs a $P e$ increase is observed with higher $K_{\|}$, due to an easier convective transport. When channel tapering is introduced, the relevance of convection largely increases for all $K_{\|}$, 
confirming the more effective mass (e.g. reactant) transport for tapered designs in under-rib areas. In Figure 5 the $P e$ field is reported on a planar section midway of $h_{G D L}$ for two cases with equal $R e, \Delta p$ and $K_{\|}$: with respect to the STD case, in the T01 design the high-Pe regime benefit extends upstream of the minimal area contraction, with convection dominated areas $(P e>1)$ more extended than diffusion-governed ones $(P e<1)$.
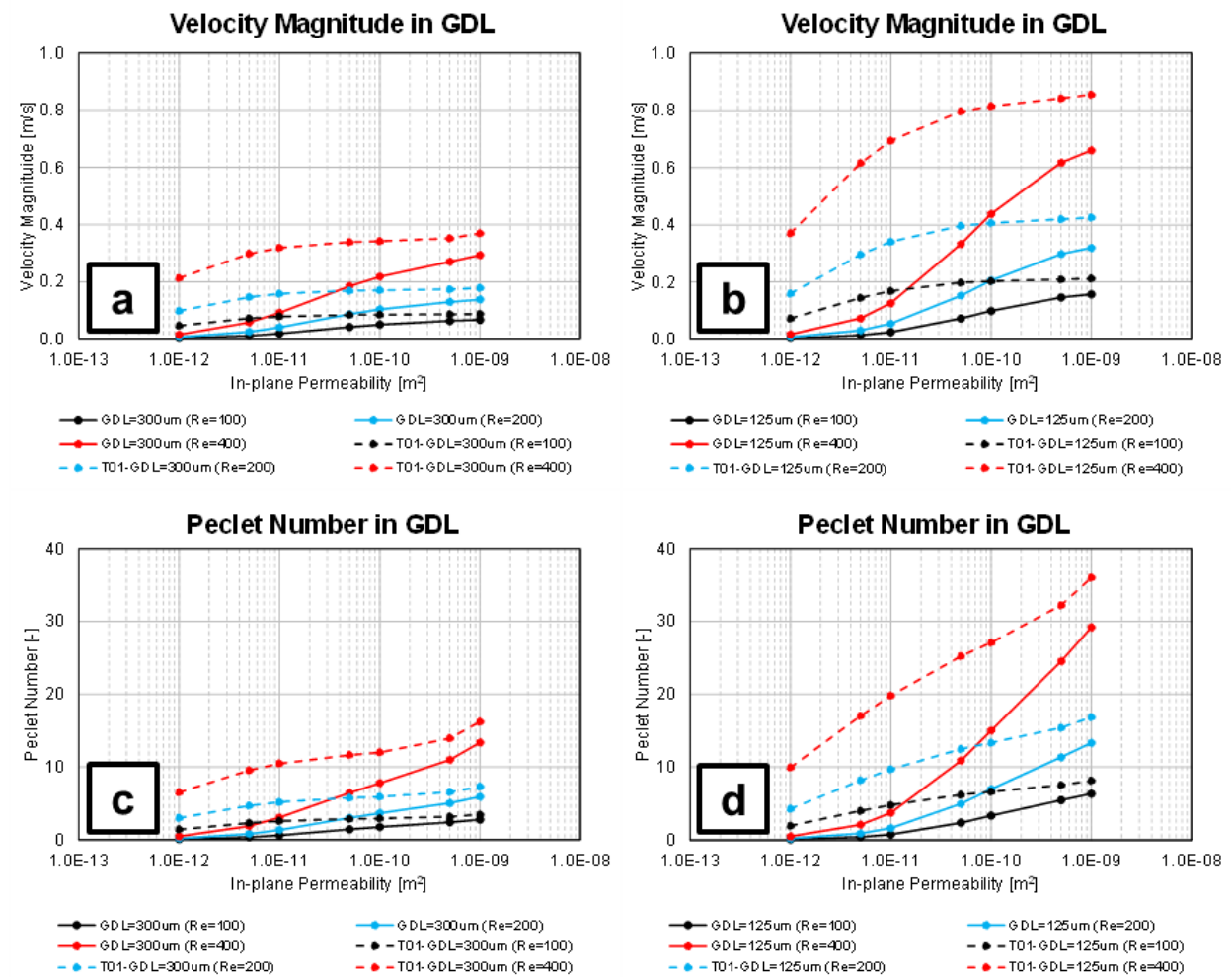

Fig. 4. Top row: surface average velocity magnitude through the GDL section for the $300 \mu \mathrm{m}$ (a) and the $125 \mu \mathrm{m}$ (b) for all cases. Bottom row: volume average $P e$ in the GDL section for the $300 \mu \mathrm{m}$ (c) and the $125 \mu \mathrm{m}$ (d) for all cases.
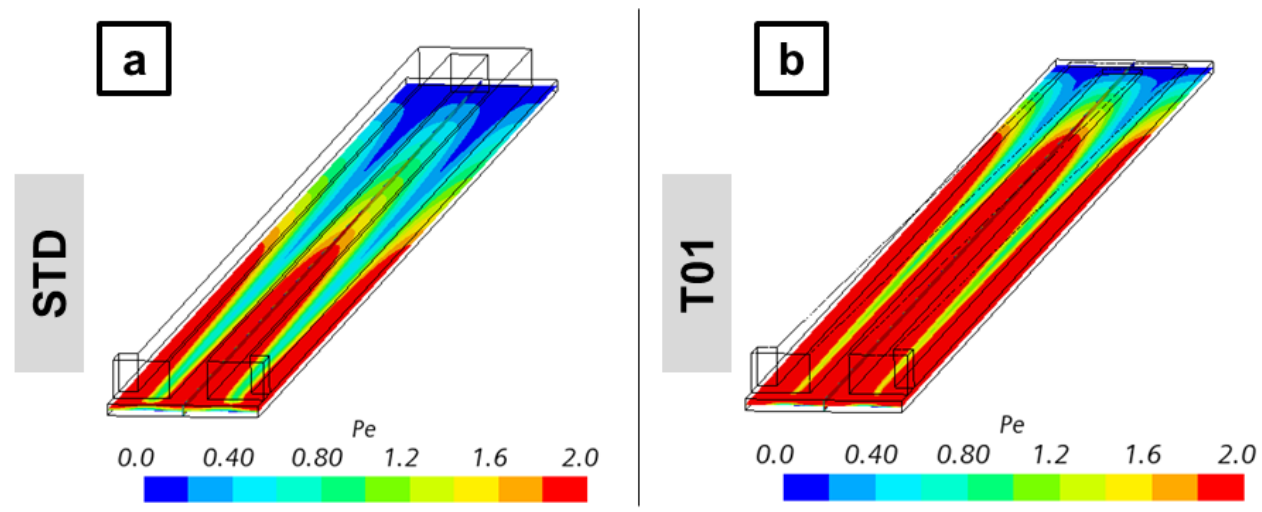

Fig. 5. Field of $P e$ on a longitudinal section midway of $h_{G D L}$ for the $R e=100$ case and $K_{\|}=$ $5 \times 10^{-11} \mathrm{~m}^{2}$ for the STD (a) and T01 (b) configuration. 
Despite the absence of electrochemically active CL, considerations can be inferred from a flow analysis regarding the limiting supply rate of reactants, ultimately determining the concentration overpotential and the maximum power density of the cell. The efficiency of mass transport on the CL surface are analysed though the wall shear stress $\left(\tau_{C L}\right)$, defined as in Eq. 2 with $\tilde{u}_{i}$ being the mean velocity along the $i$-direction, $x_{j}$ being the spatial coordinate along the $j$-direction and $\mu$ the dynamic viscosity of the fluid.

$$
\tau_{C L[P a]}=\left.\mu \frac{\partial \widetilde{u}_{i}}{\partial x_{j}}\right|_{x_{j}=0}
$$

This is used to spatially locate areas of active transport of reactants by near-wall flow convection, as well as inactive regions whose only transport mechanism is wall-normal flow diffusion. The $\tau_{C L}$ and its spatial distribution are a consequence of the GC and GDL design, hence they are used as indictors of the effectiveness of the GC/GDL system to generate mass transport to the active sites. In Figure 6 the surface-average $\tau_{C L}\left(\left\langle\tau_{C L}\right\rangle\right)$ is reported for all cases, and a generic localized maximum behaviour is observed for all cases for increasing $K_{\|}$: very low in-plane permeability limit intense CL-adjacent flow, which is allowed for higher $K_{\|}$values, although extremely high $K_{\|}$lead to a $\left\langle\tau_{C L}\right\rangle$ reduction. In Figure 7 velocity streamlines are used to explain this non-linear behaviour: after a maximum $\left\langle\tau_{C L}\right\rangle$ is reached $\left(K_{\|}=5 \times 10^{-11} \mathrm{~m}^{2}\right.$ for the STD case at $\left.R e=100\right)$, a decreasing trend of $\left\langle\tau_{C L}\right\rangle$ is observed for higher $K_{\|}$: despite more flow rate by-passes in the GDL, as testified by the velocity magnitude reduction in the GC from (a) to (c), the excessive in-plane permeability creates a minimum resistance path to the adjacent channel, thus reducing the flow interaction with the CL. In Figure 7 (bottom) this optimal trade-off is represented by the streamline interaction with the CL surface. In this context, thin GDL allow an intense flow-CL interaction, resulting in a generally higher $\left\langle\tau_{C L}\right\rangle$ and a delayed drop at high in-plane permeability. When introducing channel tapering, the forced convection leads to systematically higher $\left\langle\tau_{C L}\right\rangle$ for all cases. The relevance of it is a that a higher transport rate of reactants is created, thus effectively delaying concentration limitations, and increasing the cell power density.

In Figure 8 the field of $\tau_{C L}$ is reported for the same STD cases in Figure 6 (top row), with the analogous field for the tapered T01 design variation. Fields confirm the relevant increase in flow-CL interaction, which is quantitatively more relevant for low-medium $K_{\|}$, whereas for highly permeable GDL the gain is more limited. This agrees with the surface-average results in Figure 6, and it indicates that in the STD configuration the area of maximum $\tau_{C L}$ (hence actively supported by convective transport for the reactants supply) is located far from the channel U-bend, whereas the CL portion below the channel bend systematically observes low $\tau_{C L}$ values (only receiving reactants via a diffusive-type transport).

Aver. WSS on CL

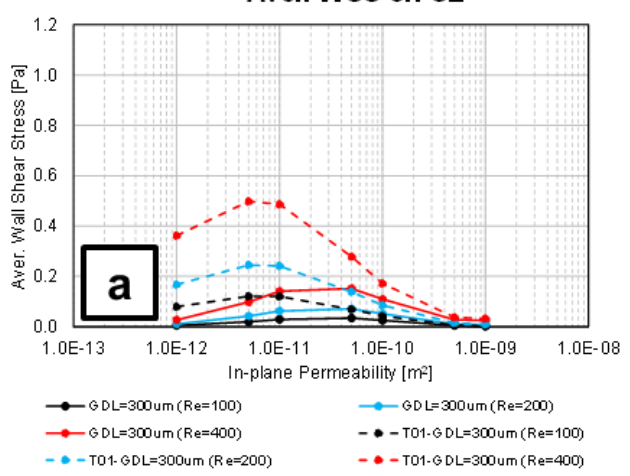

Aver. WSS on CL

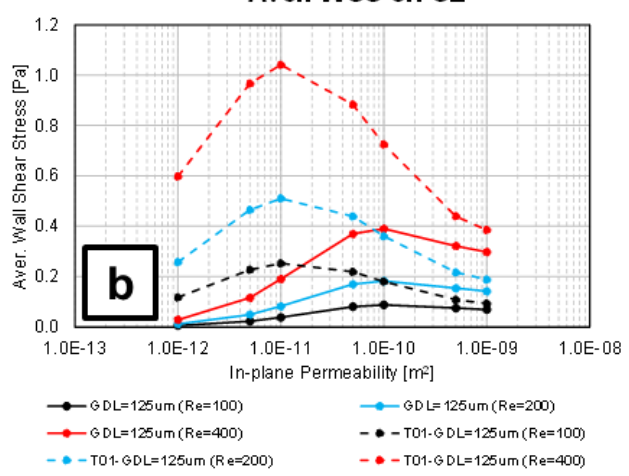


Fig. 6. Surface average of wall shear stress at CL $\left(\left\langle\tau_{C L}\right\rangle\right)$ for the $300 \mu \mathrm{m}$ (a) and the $125 \mu \mathrm{m}$ (b) for all cases.
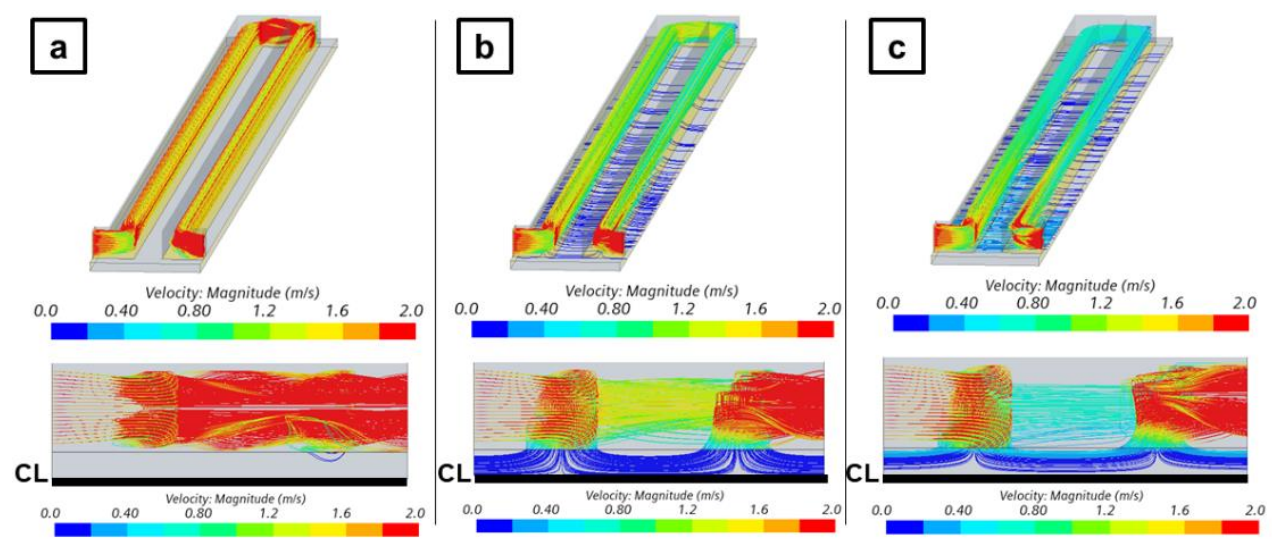

Fig. 7. Velocity streamlines for the $R e=100$ case of the STD configuration for $K_{\|}=1 \times 10^{-12} \mathrm{~m}^{2}$ (a), $K_{\|}=5 \times 10^{-11} \mathrm{~m}^{2}$ (b) and $K_{\|}=1 \times 10^{-9} \mathrm{~m}^{2}$ (c). Top: perspective view; bottom: from view.

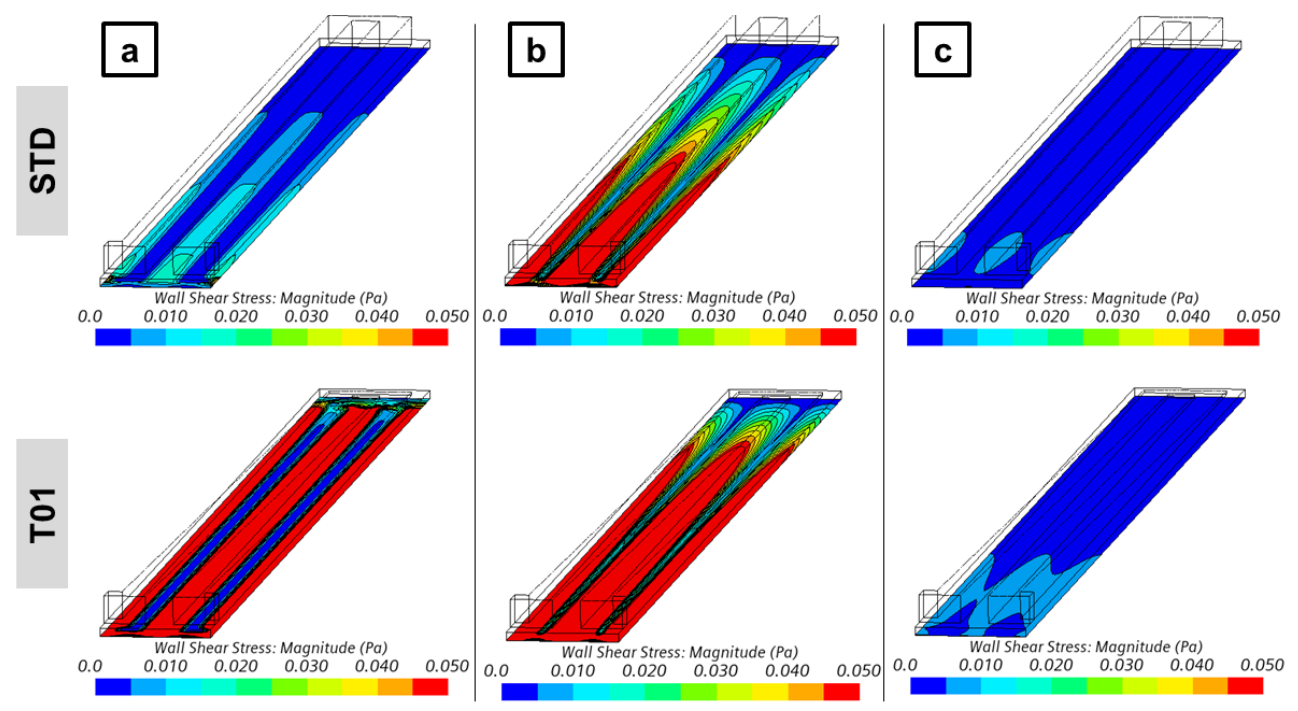

Fig. 8. Field of $\tau_{C L}$ for the $R e=100$ case for $K_{\|}=1 \times 10^{-12} \mathrm{~m}^{2}$ (a), $K_{\|}=5 \times 10^{-11} \mathrm{~m}^{2}$ (b) and $K_{\|}=$ $1 \times 10^{-9} \mathrm{~m}^{2}$ (c), for the STD (top row) and T01 (bottom row) design.

\section{Considerations on Output Power and Efficiency}

Results in Figure 8 clearly testify that the adoption of channel tapering effectively promotes the reactants-carrier flow to the $\mathrm{CL}$, thus resulting in delayed concentration overpotential and higher liming current density, which is emphasized for thin GDL designs. However, an aspect that is found critical for such design is the inclusion of pressure losses on the overall cell efficiency. Despite in multiple studies the additional pressure loss is discussed (e.g. [19]), to the authors' knowledge a comprehensive cost/benefit analysis is not undertaken to quantify whether the additional electric power produced outweighs the higher power required for fluid pumping, i.e. discussing the effect of tapering on the overall cell efficiency. In this section, a 
simplified evaluation of cell efficiency variation (with respect to a baseline configuration, which in this study is the STD case) is undertaken:

- Under the assumption that a volumetric-type compressor is used to pump reactants, the needed power $\left(P_{\text {comp }}\right)$ is proportional to the resulting pressure jump. Therefore, when moving from STD to T01 design the relative variation of $P_{\text {comp }}$ needed to overcome the higher pressure drop increases linearly with $\Delta p$, i.e. $\Delta P_{\text {comp }} \propto \Delta(\Delta p)$.

- Given the promoted reactants transport, it is hypothesized that the limiting current density increases linearly with $\left\langle\tau_{C L}\right\rangle$, leveraging a sufficient supply of reactants for electrochemical reactions. Under such assumption, the power output increases as $\Delta P_{\text {elec }} \propto \Delta \tau_{C L}$ moving from STD to T01 design.

Under the same reactant supply rate (i.e. same input chemical power), an organic-type cell efficiency can be defined as $\eta_{o}=P_{\text {elec }} / P_{\text {comp }} \propto\left\langle\tau_{C L}\right\rangle / \Delta p$. Hence, a net gain is obtained if the tapered cell efficiency $\left(\eta^{\prime}{ }_{o}\right)$ is higher than the STD one $\left(\eta_{o}\right)$. This leads to the evaluation of the $\psi$ function in Eq. 3. A net gain for the T01 design can estimated for $\psi>1$, whose values are reported in Figure 9.

$$
\psi=\frac{\eta^{\prime} o}{\eta_{o}} \propto \frac{\left\langle\tau^{\prime} C L\right.}{\left\langle\tau_{C L}\right\rangle} \cdot \frac{\Delta p}{\Delta p^{\prime}}
$$

The results show that the additional power need (negative aspect) outweighs the higher power output (positive aspect), rendering a general cell efficiency worsening with the only exception at high flow velocity $(R e=400)$ and for very low- $K_{\|} \operatorname{GDL}\left(K_{\|}=1 \times 10^{-12} \mathrm{~m}^{2}\right)$. These conclusions depict channel tapering as an effective although inefficient way to obtain more power via enforced GDL-flow, hence representing a possibility only in cases where high power density can be obtained using high-pressure reactants already available from other components, e.g. combined heat and power (CHP) cycles or turbocharged systems.
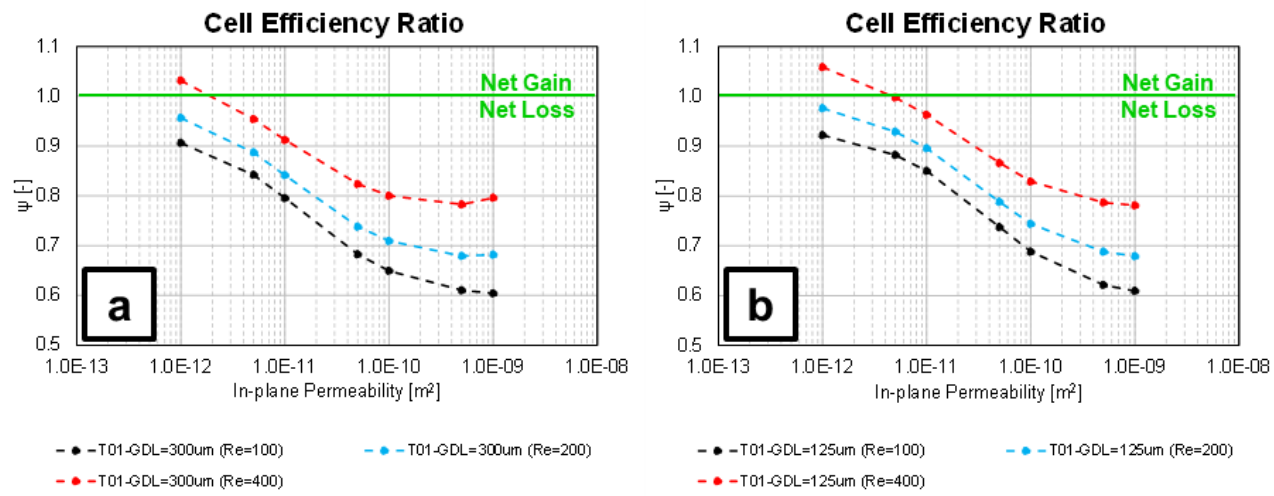

Fig. 9. Net gain/loss of efficiency using the $\psi$ function for the $300 \mu \mathrm{m}$ (a) and the $125 \mu \mathrm{m}$ (b) for all cases.

\section{Conclusions}

Polymeric Exchange Membrane Fuel Cells (PEMFC) are considered one of the most promising power propulsion systems for the decarbonization of the transportation sector. Their large-scale development and optimization are limited by an insufficient knowledge of the fluid dynamics and mass transport processes crucially limiting the electrochemistry rate under critical conditions. This is especially verified in PEMFC with serpentine-type gas distributors, for which areas in proximity of the gas channel bends are exposed to a diffusiononly reactant transport rather than a more intensive combined convective/diffusive mechanism. An engineering method to enhance the convective transport in poorly-reacting 
areas is the use of gas distributors with tapered channels, effectively forcing the flow in diffusive media and improving the reactants delivery rate and products removal.

A simulation study is presented on a section of a serpentine gas distributor, comparing conventional-type and tapered channel distributors, and evaluating the combined effect of pressure losses, catalyst layers utilization, flow regime in anisotropic diffusion media and convection/diffusion balance via a non-dimensional analysis. In order to draw general conclusions, the study is applied to 3 inlet Reynolds numbers, 7 levels of in-plane permeability for the GDL and 2 GDL thicknesses. The main outcomes of the study are:

- Channel tapering is effective in forcing a large flow percentage through the porous GDL, although this results in increased pressure losses.

- The relative contribution of convective transport grows for all cases, as confirmed by a non-dimensional $P e$ analysis. This is positive for the portion of CL under the electrode rib or close to the channel bends.

- The wall shear stress at CL is used as a local indicator of flow-CL interaction (e.g. reactants transport), and channel tapering is an effective way to substantially increase the flow at CL. This is associated to delayed concentration losses, thus allowing higher power density operation.

- An original energetic analysis based on the relative variation between the increasing power need and the additional power output reveals that a fuel cell efficiency reduction is predicted when introducing channel tapering, thus only indicating it as an effective way to increase the cell power density when high-pressure reactants are already available. This cost/benefit analysis is indicated for all the analogous type of designs variations aiming at increasing the power density of PEMFC.

\section{References}

1. J. Larminie, A. Dicks, John Wiley \& Sons, ISBN 0-470-84857-X (2003)

2. F. Barbir, Elsevier Academic Press, ISBN 978-0-12-387710-9 (2013)

3. A. d'Adamo et al., SAE Int. J. Engines 12(1):45-56, 2019, https://doi.org/10.4271/0312-01-0007.

4. G. Cicalese et al., SAE Technical Paper 2017-01-2196, 2017, https://doi.org/10.4271/2017-01-2196.

5. S. Breda, SAE Int. J. Fuels Lubr. 10(1):32-55, 2017, https://doi.org/10.4271/2017-01$\underline{0546}$

6. X. Li, I. Sabir, Int. J. of Hydrogen Energy 30 (2005), doi:10.1016/j.ijhydene.2004.09.019

7. R. Taccani, N. Zuliani, Int. J. of Hydrogen Energy 36 (2011), doi:10.1016/j.ijhydene.2010.10.026

8. J.G. Pharoah, J. of Power Sources 144 (2005), doi:10.1016/j.jpowsour.2004.11.069

9. J.P. Feser et al., J. of Power Sources 161 (2006), doi:10.1016/j.jpowsour.2006.04.129

10. C. Xu, T.S. Zhao, Elect. Communications 9 (2007), doi:10.1016/j.elecom.2006.10.031

11. S. Um, C.Y. Wang, J. of Power Sources 125 (2004), doi:10.1016/j.jpowsour.2003.07.007

12. W.M. Yan et al., J. of Power Sources 161 (2006), doi:10.1016/j.jpowsour.2006.05.007

13. M.Z. Chowdhury, Energy 161 (2018) 1, https://doi.org/10.1016/j.energy.2018.07.143

14. A. d'Adamo et al., SAE Technical Paper 2020-24-0016 (2020), https://doi.org/10.4271/2020-24-0016 
15. M. Riccardi et al., E3S Web Conf., 197 (2020), https://doi.org/10.1051/e3sconf/202019705004

16. A. d'Adamo et al., Processes, 9(3), 564 (2021); https://doi.org/10.3390/pr9030564

17. Bruggeman (1935), https://doi.org/10.1002/andp.19354160705

18. A. d'Adamo et al., Processes, 9(4), 688 (2021); https://doi.org/10.3390/pr9040688

19. Fontana et al., J. of Power Sources 234 (2013), http://dx.doi.org/10.1016/j.jpowsour.2013.01.171 